\title{
CD19CAR-CD28-CD3zeta-EGFRt-expressing Tn/mem-enriched T-lymphocytes
}

National Cancer Institute

\section{Source}

National Cancer Institute. CD19CAR-CD28-CD3zeta-EGFRt-expressing Tn/mem-enriched

T-lymphocytes. NCI Thesaurus. Code C133073.

A preparation of genetically modified lymphocytes comprised of CD62L-positive naïve and memory $T$-cells ( $\mathrm{Tn} / \mathrm{mem})$, that are transduced ex vivo with a self-inactivating (SIN) lentiviral vector expressing a hing e-optimized chimeric antigen receptor (CAR) specific for the CD19 antigen and containing CD28 and CD3 zeta signaling domains, and a truncated form of the human epidermal growth factor receptor (EGFRt), with potential immunostimulating and antineoplastic activities. Upon isolation of peripheral blood lymphocytes (PBLs), transduction of the CD62L-positive T-lymphocytes, expansion ex vivo and administration, the CD19R(EQ)-CD28-CD3zeta-EGFRt-expressing Tn/memenriched T-cells target CD19-expressing tumor cells, thereby inducing selective toxicity in CD19-expressing tumor cells. CD19 antigen is a B-cell specific cell surface antigen expressed in all B-cell lineage malignancies. Devoid of both ligand binding domains and tyrosine kinase activity, EGFRt both facilitates in vivo detection of the administered T-cells and can promote elimination of those cells upon a cetuximab-induced antibody dependent cellular cytotoxicity response. Tn/mem T-cells include naïve T-cells, central memory T-cells (T cm) and stem cell memory T-cells (T scm). CD19R(EQ) contains two point mutations in the immunog lobulin (Ig) G4 spacer region, thereby preventing recognition of the CAR by Fc receptors (FCRs). 